\title{
Dislocations in Silicon: New Results, New Challenges...
}

\section{J. Rabier}

Laboratoire de Métallurgie Physique, UMR 6630 CNRS-Université de Poitiers, SP2MI, BP 30179, F-86962 Chasseneuil Futuroscope Cedex, France

Although silicon is a model material, the basic question about the actual $\{111\}$ atomic glide plane of dislocation (shuffle set or glide set [1]) has been a matter of debate since a long time. In his pioneer work about dislocation cores in silicon, Hornstra [2], from geometrical considerations yield to the conclusion that dislocations with a Burgers vector $a / 2<110>$ are in the shuffle set. In fact, the motion of such dislocations requires the breaking of only one covalent bond as compared to the motion of dislocations in the glide set which requires the breaking of three bonds. With the advent of the weak-beam technique in the late 60's [3] it was shown that dislocations are dissociated into partials at rest [4] and also when moving [56]. This seemed to be the proof that dislocations were unambiguously gliding in the glide set. However spectroscopy measurements have shown the occurrence of point defects associated to dislocation movements even at low temperature which suggested a possible "composite" core [7].

A significant contribution to "the shuffle-glide controversy" was made by Duesbery and Joos [8]. These authors have calculated the activation energy required for double kink nucleation on a perfect dislocation in the shuffle set and on a partial dislocation in the glide set taking into account the Peierls stresses deduced from ab-initio calculations. They have shown that dissociated dislocation movement in the glide set has a lower activation energy than that of a perfect dislocation in the shuffle set in the usual investigated range of applied stresses. Another interesting point can be extracted from their calculations: extrapolating their data at high stress it can be shown that the activation energy for perfect dislocations movement in the shuffle set becomes comparable to the activation energy for dissociated dislocations in the glide set for a value of the applied stress of about $0.01 \square$, where $\square$ is the shear modulus [9].

In order to reach such high stress range, deformation techniques under confining pressure have been used. In these conditions below the usual Brittle to Ductile Transition (BDT) temperature of silicon, a different type of dislocation has been recently shown to be nucleated in silicon single crystals i.e. perfect dislocations [10], [11]. Those perfect dislocations are aligned along unusual Peierls valleys i.e. $<112>/ 30^{\circ}$ and $<123>/ 41^{\circ}$. Another striking feature in these deformation substructures is the zigzag configurations of dislocations that demonstrates the existence of very strong pinning points. Although no direct evidence has been put forward that those perfect dislocations are located in the shuffle set, the atomistic calculations of core structures [12] and nucleation events of dislocations at high stress [13] as well as experimental observations are consistent with those perfect dislocations belonging to the shuffle set.

Beside the challenge of imaging $<112>/ 30^{\circ}$ dislocation core with sub-Angstrom resolution (dumbbells viewed along $<112>$ are 78 pm apart), several questions can be addressed from these observations. Are perfect shuffle dislocation reconstructed? Are the occurrence of 
$<123>/ 41^{\circ}$ apparent Peierls valleys resulting from reconstructed kinks? Is the BDT related to the change in core structure of dislocations [14]?

\section{References}

[1] J.P. Hirth and J. Lothe, Theory of Dislocations. McGraw-Hill: New York, (1968) 353.

[2] J. Hornstra, J. Phys. Chem. Sol. 5 (1958) 129.

[3] D.J.H. Cockayne, I.L.F. Ray, and M.J. Wheelan, Phil. Mag. A20 (1969) 1265.

[4] I.L.F. Ray, and D.J.H. Cockayne, Phil. Mag. 22 (1970) 853.

[5] R. Meingast, and H. Alexander, phys. stat. sol. (a)17 (1973) 229.

[6] A. Gomez, D.J.H. Cockayne, P.B. Hirsch and V. Vitek, Phil. Mag. 31 (1975) 105.

[7] H. Alexander, Dislocations in Solids, vol. 7. F.R.N. Nabarro, Elsevier, North Holland, (1986) 224.

[8] M.S. Duesbery and B. Joos, Phil. Mag. A 74 (1996) 253.

[9] J. Rabier and J.L Demenet, phys. stat. sol. (a) 222 (2000) 63.

[10] J. Rabier, P. Cordier, J.L. Demenet and H. Garem, Mater. Sc. \& Eng. A 309-310 (2001) 74.

[11] J. Rabier and J.L. Demenet, Scripta Mater. 45 (2001) 1259.

[12] J. Goddet, L. Pizzagalli, S. Brochard and P. Beauchamp, PRB 70 (2004) 054109.

[13] L. Pizzagalli, P. Beauchamp and J. Rabier, Phil. Mag A. 83 (2003) 1191.

[14] The author acknowledges his colleagues at LMP, P. Beauchamp, J.L. Demenet and L. Pizzagalli for stimulating discussions.

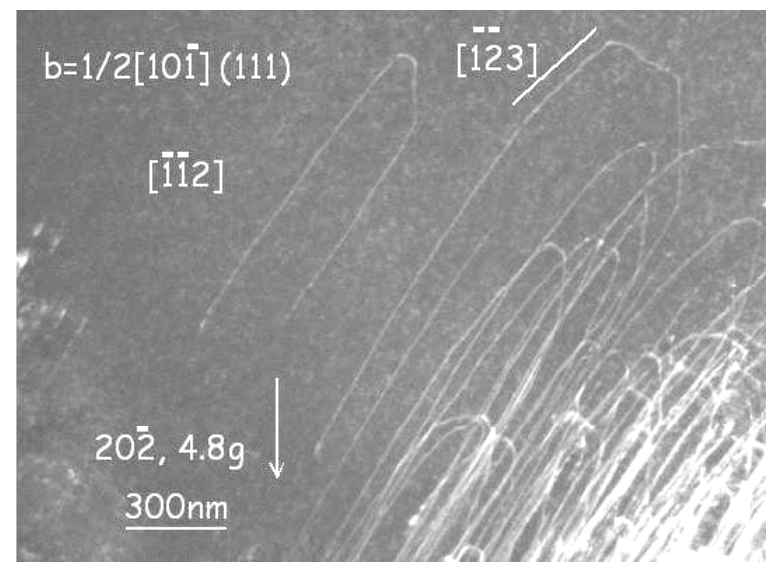

(a)

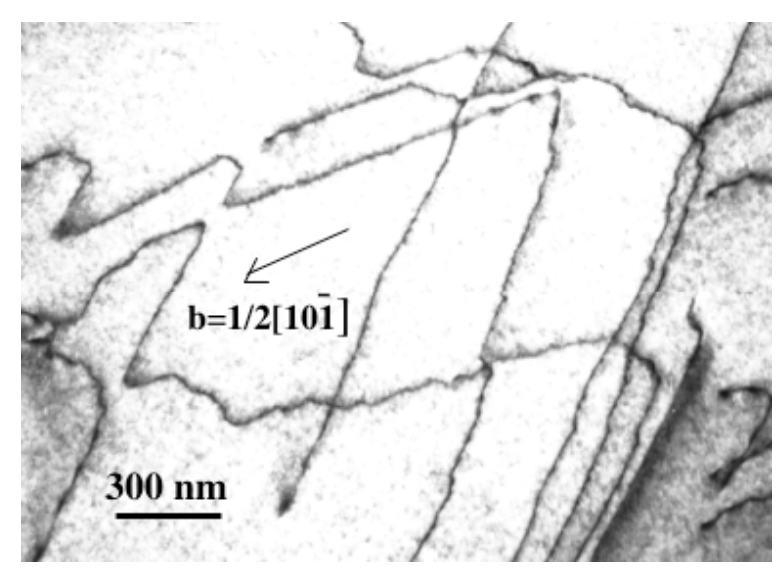

(b)

Fig.1. FZ silicon single crystal deformed under a $5 \mathrm{GPa}$ hydrostatic pressure and an applied uniaxial stress at different temperatures

(a) Room temperature: perfect dislocations with $<112>/ 30^{\circ}$ and $<123>/ 41^{\circ}$ Peierls valleys

(b) $150^{\circ} \mathrm{C}$ : perfect dislocations with screw and $<112>/ 30^{\circ}$ Peierls valleys showing strong pinning points 